\title{
ACCURATE BENDING STRENGTH ANALYSIS OF ASYMMETRIC GEARS USING THE NOVEL ES-PIM WITH TRIANGULAR MESH
}

\author{
S. Wang ${ }^{1}$, G. R. Liu ${ }^{1,2}$, G. Y. Zhang ${ }^{2}$ and L. Chen ${ }^{1}$ \\ ${ }^{1}$ Centre for Advanced Computations in Engineering Science (ACES), Department of \\ Mechanical Engineering, National University of Singapore (NUS), 9 Engineering \\ Drive 1, 117576, Singapore. Tel: +0065-65164797 \\ Email: g0800348@nus.edu.sg \\ ${ }^{2}$ Singapore-MIT Alliance (SMA), E4-04-10, 4 Engineering Drive 3, 117576, Singapore
}

\begin{abstract}
This paper extends the edge-based smoothed point interpolation method (ES-PIM) to bending strength analysis of asymmetric gears with complex outlines. Five sets of asymmetric gears with pressure angles of $20^{\circ} / 20^{\circ}, 25^{\circ} / 20^{\circ}, 30^{\circ} / 20^{\circ}, 35^{\circ} / 20^{\circ}$ and $40^{\circ} / 20^{\circ}$ were generated by a specially designed rack cutter. Four key factors, e.g. accuracy, convergence, the convergence rate and the computational efficiency of the present ES-PIM were checked in great detail on these five models, and the distributions of bending stresses at the fillet of the drive side were carefully investigated. The finite element method (FEM) was also used to calculate the abovementioned factors to stress the advantages of ES-PIM. The numerical results indicate that ES-PIM can provide more efficient and accurate solutions in the stress field than the FEM, and is very suitable for stress analysis of complicated asymmetric gears.
\end{abstract}

Keywords: Asymmetric gear; Bending strength; Accurate stress; Numerical method; Point interpolation method (PIM); Smoothing operation.

\section{INTRODUCTION}

Gear transmission is one of the most important mechanical transmissions in engineering systems, so its reliability is essential. However, fracture always occurs in gear teeth due to their low bending strength, which leads to invalidation and thus failure of the entire transmission (Bible et al., 1994). To avoid this failure, several methods have been used to improve the bending strength of gear teeth. Among these, modifying the shape of the tooth is an effective and widely used method (Bible et al., 1994; Cavdar et al., 2005). To date, several kinds of asymmetric gears have been developed. Deng et al. (2003) made changes to the pressure angle on the coast side to increase the bending stiffness of the tooth. Kapelevich (2000) and Kapelevich and Kleiss (2002) have suggested using a larger pressure angle on the drive side and a fixed pressure angle on the coast side. With a larger pressure angle on the drive side, Litvin 
et al. (2000) proposed a modified geometry of an asymmetric spur gear drive designed as a combination of an involute gear and a double crowned pinion. Muni et al. (2007) and Kumar et al. (2008) used a direct gear design method for the optimisation of bending strength of asymmetric spur gear drives. Xiao et al. (2006), Xiao et al. (2008) and Xiao (2008) have used a non-stranded asymmetric rack cutter to generate the gear pair via a series of analyses on bending stresses, vibration, thermal conduction, etc. All these researches have put forward a series of solutions to improve the load carrying ability of asymmetric gears.

During gear transmission, the teeth withstand tensile stresses on the loaded side (drive side) and compressive stresses on the opposite side (coast side). Tooth fracture always occurs at the fillet of the tensile-stress side (drive side), where there is also a stress concentration. So, it is essential to carry out accurate bending stress analysis at the fillet of the tensile-stress side (drive side) in order to check the strength of the teeth (Wang, 2003). Among these stresses at the fillet, the maximum bending stress of the tooth has a significant impact on fracturing and should be carefully checked. Examination of run-in teeth reveals that when a single tooth carries the full load and the load is applied at the highest point of single tooth contact (HPSTC), the maximum bending stress occurs (Wang, 2003). Therefore, when performing bending stress analysis, the load is always applied at the HPSTC.

In the literature, the bending strength analysis of the gear is always dealt with as a 2-D problem, and the finite element method (FEM) is utilised to perform the analysis (Bible et al., 1994; Cavdar et al., 2005; Deng et al., 2003). Since the linear triangular element can be generated efficiently without manual operation, even for complicated domains, it is quite suitable for the gear problems with complex outlines. However, because of the well-known overly-stiff behaviour of the fully compatible displacement of the FEM model based on the Galerkin weak form, the FEM using linear triangular elements usually gives poor solutions, especially for stress components. Recently, a generalised gradient smoothing technique, employing a definition of $\mathrm{G}$ space which includes both continuous and discontinuous functions and the notion of a weakened weak $\left(\mathrm{W}^{2}\right)$ formulation, also called the Generalised Smoothed Galerkin (GS-Galerkin) weak form, has been developed by Liu (2008, 2010). The $\mathrm{W}^{2}$ formulation is the foundation of a series of novel and effective numerical methods which can effectively overcome the problems discussed previously (Liu and Zhang, 2008). Compared to the compatible FEM model, these methods behave more softly and possess a number of attractive properties according to the formation of smoothing domains (Liu and Zhang, 2008; Liu, 2008, 2010).

The edge-based smoothed point interpolation method (ES-PIM) is one of these novel methods, in which the strains are smoothed over the smoothing domains associated with the edges of background triangles (Liu and Zhang, 2008; Liu et al., 2008). The polynomial and/or radial PIM shape functions, which possess the Kronecker delta property and hence facilitate the treatment of essential boundary conditions (Liu, 2002), are used to construct displacement fields in this method. Based 
on the triangular elements, four schemes of selecting support nodes for creating PIM (polynomial or radial) shape functions have been proposed, and four models have been developed, which are the ES-PIM of T3-scheme (ES-PIM (T3)), the ES-PIM of T6/3-scheme (ES-PIM (T6/3)), the ES-RPIM of T6-scheme (ES-RPIM (T6)) and the ES-RPIM of T2L-scheme (ES-RPIM (T2L)). The T-scheme can solve not only the singularity problem encountered in constructing polynomial PIM shape functions, but also improves the efficiency of the numerical methods (Liu and Zhang, 2008). Numerical results have demonstrated that the ES-PIM models have a close-to-exact stiffness and produce "super-convergence" and "ultra-accurate" solutions (Liu and Zhang, 2008; Liu et al., 2008; Liu, 2002). Most importantly, the ES-PIM (T3) model has been found to achieve the highest computational efficiency compared to the other ES-PIM models and the linear FEM model. However, the conclusions described above were drawn based on problems with simple geometries, e.g. beam and plate. The efficiency of this method in terms of problems with complex geometries has not been carefully checked yet.

This work just extends the ES-PIM (T3) method to the bending strength analysis of an asymmetric gear which possesses very complex outlines. First, the basic principles of the ES-PIM (T3) method are introduced briefly. Then, based on a specially designed rack cutter, five different asymmetric gear models were generated, with a greater pressure angle $\left(20^{\circ}, 25^{\circ}, 30^{\circ}, 35^{\circ}, 40^{\circ}\right)$ on the drive side and a normal pressure angle $\left(20^{\circ}\right)$ on the coast side. Third, some important ES-PIM (T3) properties, e.g. computational accuracy in the stress field, convergence of strain energy, the convergence rate of the strain energy norm and computational efficiency were checked in great detail and compared with the FEM-T3 method. Finally, the bending stress distributions at the fillet of the drive side were studied. From this analysis, it can be seen that the ES-PIM (T3) method is one of "super-convergence", and provides more efficient and accurate solutions in the stress field than the FEM with the same coarse triangular mesh. The ES-PIM (T3) method is quite suitable for bending strength analysis of complicated asymmetric gear problems with complex outlines and is capable of dealing with stress concentration problems.

\section{THE ES-PIM (T3) IN BRIEF}

The main differences in numerical implementation between the ES-PIM (T3) and FEM are the calculation of the strain field and the procedure of numerical integration. A brief illumination of the differences is presented in this section.

\section{Displacement Field Approximation Using PIM}

The point interpolation method (PIM) obtains an approximation by letting the interpolation function pass through the function values at each scattered node within the local supporting domain. In total, there are two types of PIM that have been 
developed using different basis functions, i.e., polynomial PIM using polynomial basis functions (Liu and $\mathrm{Gu}, 2001$ ) and radial PIM (RPIM) using radial basis functions (Wang and Liu, 2002).

For polynomial PIM, the formulations start with the following assumption:

$$
\mathbf{u}(\mathbf{x})=\sum_{i=1}^{n} p_{i}(\mathbf{x}) a_{i}=\mathbf{p}^{\mathrm{T}}(\mathbf{x}) \mathbf{a}
$$

where $\mathbf{u}(\mathbf{x})$ is a field variable function defined in the Cartesian coordinate space; $p_{i}(\mathbf{x})$ is the basis function of monomials which is usually built utilising the Pascal's triangles; $a_{i}$ is the corresponding coefficient and $n$ is the number of nodes in the local support domain.

The complete polynomial basis of orders 1 and 2 can be written as:

$$
\begin{aligned}
& \mathbf{p}^{\mathrm{T}}(\mathbf{x})=\left\{\begin{array}{lll}
1 & x & y
\end{array}\right\} \text { Basis of complete first order } \\
& \mathbf{p}^{\mathrm{T}}(\mathbf{x})=\left\{\begin{array}{llllll}
1 & x & y & x^{2} & x y & y^{2}
\end{array}\right\} \quad \text { Basis of complete second order }
\end{aligned}
$$

For radial PIM, using radial basis functions augmented with polynomials, the field function can be approximated as follows:

$$
\mathbf{u}(\mathbf{x})=\sum_{i=1}^{n} r_{i}(\mathbf{x}) a_{i}+\sum_{j=1}^{m} p_{j}(\mathbf{x}) b_{j}=\mathbf{r}^{\mathrm{T}}(\mathbf{x}) \mathbf{a}+\mathbf{p}^{\mathrm{T}}(\mathbf{x}) \mathbf{b}
$$

where $r_{i}(\mathbf{x})$ and $p_{j}(\mathbf{x})$ are the radial basis functions and polynomial basis functions, respectively; $a_{i}$ and $b_{j}$ are the corresponding coefficients; $n$ is the number of nodes in the local support domain and $m$ is the number of polynomial terms.

In the ES-PIM (T3), the linear polynomial PIM is used to approximate the displacement field and form the global stiffness matrix. The coefficients in Eq. (4) can be determined by enforcing the field function to be satisfied at the $n$ nodes within the local support domain.

$$
\mathbf{a}=\mathbf{P}^{-1} \mathbf{u}_{s}
$$

where $\mathbf{P}$ is the moment matrix; for a 2-D linear integration problem, $\mathbf{P}$ can be expressed as:

$$
\mathbf{P}=\left[\begin{array}{lll}
1 & x_{1} & y_{1} \\
1 & x_{2} & y_{2} \\
1 & x_{3} & y_{3}
\end{array}\right]_{3 \times 3}
$$

$\mathbf{u}_{s}$ is the displacement component vector at all the $n$ nodes of the support domain:

$$
\mathbf{u}_{s}^{T}=\left[\begin{array}{llll}
u_{1 x} & u_{1 y} \cdots u_{n x} & u_{n y}
\end{array}\right]_{1 \times 2 n}
$$

Note that $\mathbf{P}^{-1}$ may not exist. Approaches dealing with this problem can be found in the literature (Liu, 2002). Therefore, we just consider that the moment matrix is invertible. 
Substituting Eq. (6) into Eq. (5), we can obtain:

$$
\mathbf{u}(\mathbf{x})=\sum_{i=1}^{n} \varphi_{i}(\mathbf{x}) u_{i}=\mathbf{\Phi}^{\mathrm{T}}(\mathbf{x}) \mathbf{u}_{s}
$$

where $\varphi_{i}(\mathbf{x})$ is the shape function which processes the Kronecker delta property (Wang and Liu, 2002) and is defined by (Liu and Quek, 2003):

$$
\varphi_{i}(\mathbf{x})=\mathbf{p}^{\mathrm{T}}(\mathbf{x})_{i j} \mathbf{P}_{j i}^{-1}
$$

In the above formula, it is noted that we need to properly select $n$ nodes for interpolation, thus ensuring a non-singular moment matrix (Liu, 2002). The next subsection will focus on the principles of selecting nodes.

\section{Cell-based T-scheme for Node Selection}

The problem domain is first discretised with three-noded triangular cells (elements). In the frame work of ES-PIM (T3), a linear displacement field is constructed within each triangular cell, which is exactly the same as that in the standard FEM. As illustrated in Figure 1a, no matter the point of interest $x$ located in an interior element (element $i$ ) or a boundary element (element $j$ ), only the three nodes of the corresponding cell ( $i 1-i 3$ or $j 1-j 3)$ are selected.

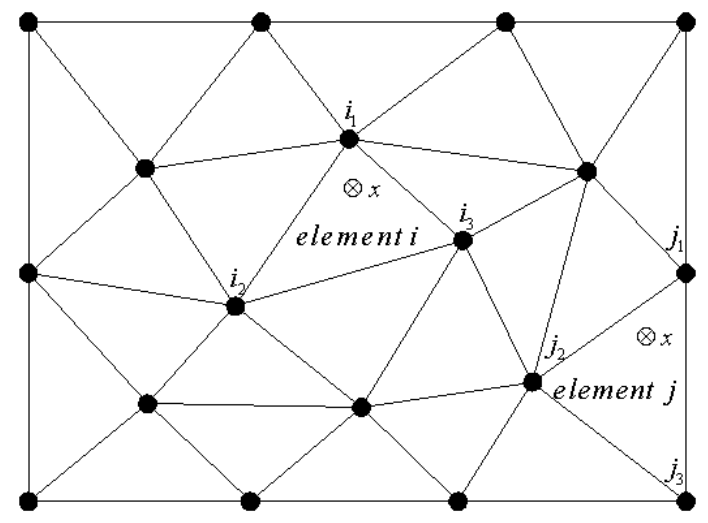

(a) Node selection

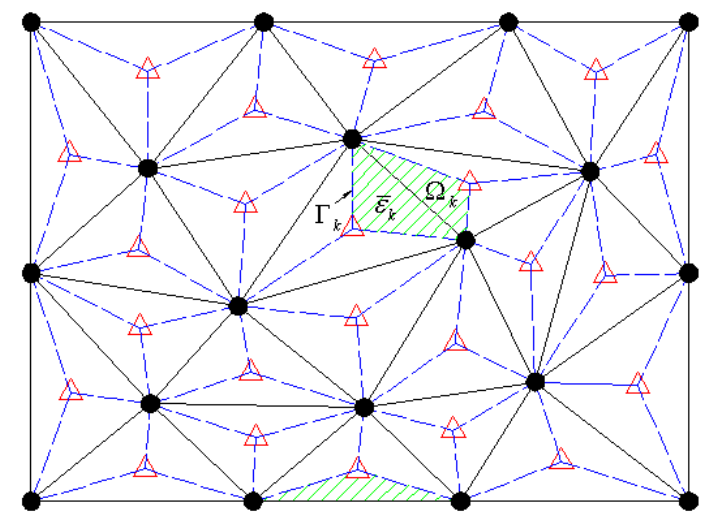

(b) Construction of smoothing domains

Figure 1. Illustration of the T3-scheme

\section{Edge-based Smoothed Strains}

In the framework of the $\mathrm{W}^{2}$ formula, the gradient of the field function (strains) will be obtained using the following generalised smoothing operation which considers both continuous and discontinuous displacement functions (Liu and Zhang, 2008; Liu et al., 2005). 


$$
\overline{\boldsymbol{\varepsilon}}_{k}= \begin{cases}\frac{1}{A_{k}} \int_{\Omega_{k}} \tilde{\boldsymbol{\varepsilon}}(\mathbf{x}) \mathrm{d} \Omega_{k}=\frac{1}{A_{k}} \int_{\Gamma_{k}} \mathbf{L}_{n} \mathbf{u}(\mathbf{x}) \mathrm{d} \Gamma_{k} & \text { when } \mathbf{u}(\mathbf{x}) \text { is continuous in } \Omega_{k} \\ \frac{1}{A_{k}} \int_{\Gamma_{k}} \mathbf{L}_{n} \mathbf{u}(\mathbf{x}) \mathrm{d} \Gamma_{k} & \text { when } \mathbf{u}(\mathbf{x}) \text { is discontinuous in } \Omega_{k}\end{cases}
$$

where $\tilde{\boldsymbol{\varepsilon}}$ is the compatible strain obtained by:

$$
\tilde{\boldsymbol{\varepsilon}}=\mathbf{L}_{\mathrm{d}} \mathbf{u}(\mathbf{x}) \text { where } \mathbf{L}_{\mathrm{d}}\left(\frac{\partial}{\partial x}, \frac{\partial}{\partial y}\right)=\left[\begin{array}{cc}
\frac{\partial}{\partial x} & 0 \\
0 & \frac{\partial}{\partial y} \\
\frac{\partial}{\partial y} & \frac{\partial}{\partial x}
\end{array}\right]_{3 \times 2}
$$

where $\mathbf{L}_{n}$ is the matrix of unit outward normal; $\overline{\boldsymbol{\varepsilon}}_{k}$ is the smoothed strain over the smoothing domain $\Omega_{k}$ and $A_{k}=\int_{\Omega_{k}} \mathrm{~d} \Omega_{k}$ is the area of $\Omega_{k}$.

To perform generalised strain smoothing, the problem domain is first discretised using three-noded triangular elements and then the stationary and non-overlapping smoothing domains are constructed based on these triangles, such that $\Omega=\Omega_{1} \cup \Omega_{2} \cup \cdots \cup \Omega_{N_{s}}$ and $\Omega_{i} \cap \Omega_{j}=\varnothing, i \neq j$ in which $N_{s}$ is the number of smoothing domains. For the ES-PIM (T3), smoothing domains are constructed with respect to the edges of the triangular elements by connecting two ends of an edge to the centroids of two adjacent cells, as illustrated in

Figure 1b. Thus, the number of smoothing domains $\left(N_{s}\right)$ equals the number of edges of triangles $\left(N_{\text {edge }}\right)$.

Substituting Eq. (2) into Eq. (1), the edge-based smoothed strain, $\overline{\boldsymbol{\varepsilon}}_{k}$, can now be written in the following matrix form of nodal displacements:

$$
\overline{\boldsymbol{\varepsilon}}_{k}=\frac{1}{A_{k}} \int_{\Gamma_{k}} \mathbf{L}_{n} \boldsymbol{\Phi}^{\mathrm{T}}(\mathbf{x}) \mathbf{u}_{s} \mathbf{d} \Gamma_{k}=\sum_{i \in N_{i n f l}} \overline{\mathbf{B}}_{i}\left(\mathbf{x}_{k}\right) \mathbf{u}_{s i}
$$

where $\Phi$ is the matrix of PIM shape functions; $N_{\text {infl }}$ is the number of field nodes involved in constructing the smoothed strain fields within $\Omega_{k}$ and $\overline{\mathbf{B}}_{i}\left(\mathbf{x}_{k}\right)$ is termed as the smoothed strain matrix that can be expressed as:

$$
\overline{\mathbf{B}}_{i}\left(\mathbf{x}_{k}\right)=\left[\begin{array}{cc}
\bar{b}_{i x}\left(\mathbf{x}_{k}\right) & 0 \\
0 & \bar{b}_{i y}\left(\mathbf{x}_{k}\right) \\
\bar{b}_{i y}\left(\mathbf{x}_{k}\right) & \bar{b}_{i x}\left(\mathbf{x}_{k}\right)
\end{array}\right]_{3 \times 2}
$$


where

$$
\bar{b}_{i l}\left(\mathbf{x}_{k}\right)=\frac{1}{A_{k}} \int_{\Gamma_{k}} \varphi_{i}\left(\mathbf{x}_{k}\right) n_{l}\left(\mathbf{x}_{k}\right) \mathrm{d} \Gamma_{k} \quad(l=x, y)
$$

Using a Gauss integration scheme, the above integration can be further expressed as follows:

$$
\bar{b}_{i l}\left(\mathbf{x}_{k}\right)=\frac{1}{A_{k}} \sum_{m=1}^{N_{\text {seg }}}\left[\sum_{n=1}^{N_{\text {gau }}} w_{n} \varphi_{i}\left(\mathbf{x}_{m n}\right) n_{i}\left(\mathbf{x}_{m}\right)\right] \quad(l=x, y)
$$

where $N_{\text {seg }}$ is the number of segments of the boundary $\Gamma_{k} ; N_{\text {gau }}$ is the number of Gauss points located in each segment on $\Gamma_{k} ; w_{n}$ is the corresponding weight number of Gauss integration scheme. As linear shape functions are used in this work, $N_{\text {gau }}=1$ is adopted to perform the integration.

\section{Discretised System Equations}

For the ES-PIM models, the following generalised smoothed Galerkin (GS-Galerkin) weak form is used to derive the discretised system equations (Liu, 2008, 2010):

$$
\int_{\Omega} \delta(\overline{\boldsymbol{\varepsilon}}(\mathbf{u}))^{\mathrm{T}} \mathbf{D}(\overline{\boldsymbol{\varepsilon}}(\mathbf{u})) \mathrm{d} \Omega-\int_{\Omega} \delta \mathbf{u}^{\mathrm{T}} \mathbf{b} \mathrm{d} \Omega-\int_{\Gamma} \delta \mathbf{u}^{\mathrm{T}} \mathbf{t}_{\Gamma} \mathrm{d} \Gamma=0
$$

which has exactly the same form as the standard Galerkin weak form. Thus, the formulation procedure is exactly the same as that in the standard FEM, and all we need to do is to use the edge-based smoothed strain $\overline{\boldsymbol{\varepsilon}}$ in place of the compatible strain $\tilde{\boldsymbol{\varepsilon}}$.

The overall procedure of the presented methods is as follows. First, the displacement field will be constructed by using PIM. Following this, the smoothed strains $\overline{\boldsymbol{\varepsilon}}$ will be obtained using Eq. (3). Finally, by substituting the assumed displacements and the smoothed strains into the generalised Galerkin weak form (Eq. (7)), and invoking the arbitrary nature of the variation operations, a set of discretised algebraic system equations can be obtained in the following matrix form:

$$
\overline{\mathbf{K}} \mathbf{u}=\mathbf{f}
$$

where $\mathbf{f}$ is the force vector that can be obtained as:

$$
\mathbf{f}=-\int_{\Omega} \boldsymbol{\Phi}^{\mathrm{T}} \mathbf{b} \mathrm{d} \Omega+\int_{\Gamma} \boldsymbol{\Phi}^{\mathrm{T}} \mathbf{t}_{\Gamma} \mathrm{d} \Gamma
$$

and the stiffness matrix $\overline{\mathbf{K}}$ is assembled from the sub-stiffness matrix for all the integration cells, which are exactly the edge-based smoothing domains for the present method.

$$
\overline{\mathbf{K}}_{i j}=\sum_{k=1}^{N_{s}} \overline{\mathbf{K}}_{i j(k)}=\sum_{k=1}^{N_{s}} A_{k} \overline{\mathbf{B}}_{i}^{T} \mathbf{D} \overline{\mathbf{B}}_{j}
$$


where the smoothed strain matrixes are obtained using Eq. (4).

Substituting the stiffness matrix $\overline{\mathbf{K}}$ into the static system of equations:

$$
\overline{\mathbf{K}} \mathbf{U}=\mathbf{F}
$$

where $\mathbf{F}$ is the equivalent force vector.

The vector $\mathbf{U}$ of the displacements at all the nodes thus can be calculated by Eq. (11) based on any methods, e.g. Gauss elimination, LU decompositions and iterative methods (Liu and Quek, 2003). Finally, the interested variables, e.g. stresses and strains in the field, can be calculated based on the vector $\mathbf{U}$ following standard procedures used as in the FEM.

\section{ESTABLISHMENT OF ASYMMETRIC GEAR MODELS}

\section{Formulation of the Asymmetric Gear}

In this section, five asymmetric gear models, each of which possesses a greater pressure angle on the drive side and a normal pressure angle on the coast side, were generated by a specially designed rack cutter. Figure 2 presents the schematic of the specially designed rack cutter which uses a larger pressure angle on one side and a normal pressure angle on the opposite side. Only one round corner where there is only one radius $(\rho)$ is designed at the top of the rack cutter as the connection of two straight outlines of the rack to ensure that the fillet of the generated asymmetric gears is trochoidal. The design parameters of the rack cutter are listed in Table 1.

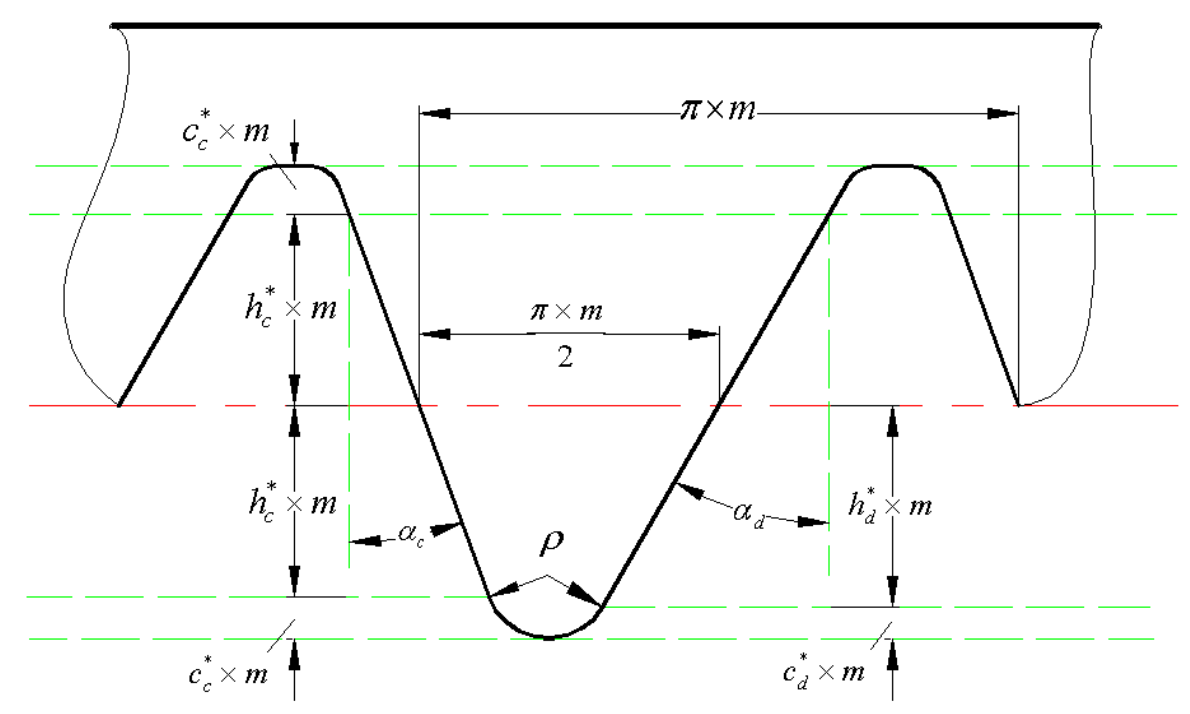

Figure 2. Profile of the specially designed rack cutter with one fillet at the tip 
Table 1. Parameters of the specially designed rack cutter

\begin{tabular}{lll}
\hline Parameter & Symbol & Quantitative value \\
\hline Module & $m$ & $5 \mathrm{~mm}$ \\
Pressure angle on the coast side & $\alpha_{c}$ & $20^{\circ}$ \\
Pressure angles on the drive side & $\alpha_{d}$ & $20^{\circ} / 25^{\circ} / 30^{\circ} / 35^{\circ} / 40^{\circ}$ \\
Addendum coefficient on the coast side & $h_{c}^{*}$ & 1 \\
$\begin{array}{l}\text { \#1 Tip radius } \\
\text { \#2 Bottom clearance coefficient on the }\end{array}$ & $c_{c}^{*}$ & $c_{c}^{*}=\frac{\rho \times\left(1-\sin \alpha_{c}\right)}{m}$ \\
$\begin{array}{l}\text { coast side } \\
\text { \#3 Bottom clearance coefficient on the }\end{array}$ & $c_{d}^{*}$ & $c_{d}^{*}=\frac{\rho \times\left(1-\sin \alpha_{d}\right)}{m}$ \\
$\begin{array}{l}\text { drive side } \\
\text { \#4 Addendum coefficient on the drive side }\end{array}$ & $h_{d}^{*}$ & $h_{d}^{*}=h_{c}^{*}+c_{c}^{*}-c_{d}^{*}$ \\
\hline
\end{tabular}

$\# 1,2,3,4$ For different $\alpha_{d}$, the quantitative values of $\rho, c_{c}^{*}, c_{d}^{*}, h_{d}^{*}$ are different, and are listed in Table 2.

Table 2. Quantitative values of $\rho, c_{c}^{*}, c_{d}^{*}, h_{d}^{*}$ associated with special $\alpha_{d}$ Unit: $\mathrm{mm}$

\begin{tabular}{llllll}
\hline$\alpha_{d}$ & $\mathbf{2 0}^{\circ}$ & $\mathbf{2 5}^{\circ}$ & $\mathbf{3 0}^{\circ}$ & $\mathbf{3 5}^{\circ}$ & $\mathbf{4 0}^{\circ}$ \\
\hline$\rho$ & 2.2424 & 1.9657 & 1.6592 & 1.3186 & 0.9390 \\
$c_{c}^{*}$ & 0.2951 & 0.2587 & 0.2183 & 0.1735 & 0.1236 \\
$c_{d}^{*}$ & 0.2951 & 0.2270 & 0.1659 & 0.1125 & 0.0671 \\
$h_{d}^{*}$ & 1 & 1.0317 & 1.0524 & 1.0611 & 1.0565 \\
\hline
\end{tabular}

Based on envelope principles (Fetvaci and Imrak, 2008; Su and Houser, 2000), the outlines of five asymmetric gear models can be drawn as shown in Figure 3. The parameters of these five gear models are list in Table 3. Note that the maximum bending stress occurs at the time when a single tooth carries the full load. Therefore, 
the selection of one single tooth contact is enough for an investigation into the maximum bending stress (Wang, 2003).

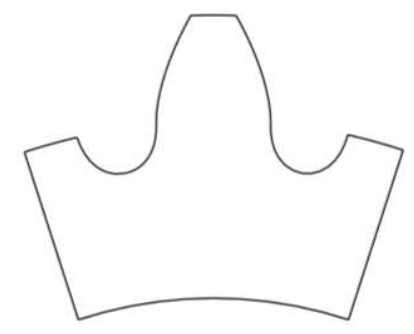

a) Model 1 with pressure angles $20^{\circ} / 20^{\circ}$

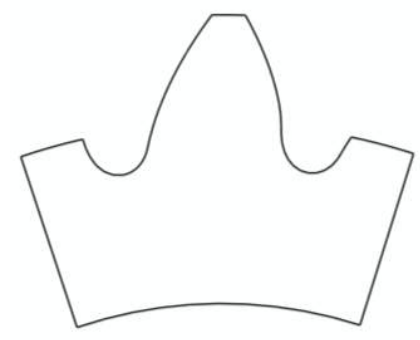

c) Model 3 with pressure angles $30^{\circ} / 20^{\circ}$

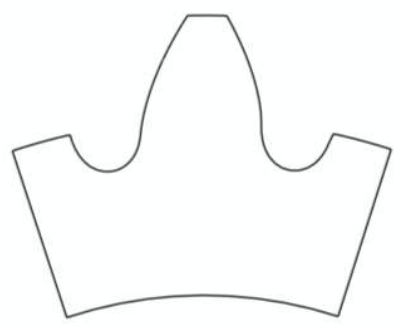

b) Model 2 with pressure angles $25^{\circ} / 20^{\circ}$

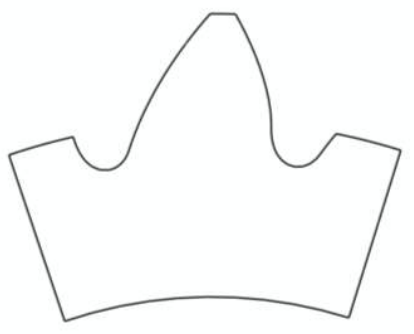

d) Model 4 with pressure angles $35^{\circ} / 20^{\circ}$

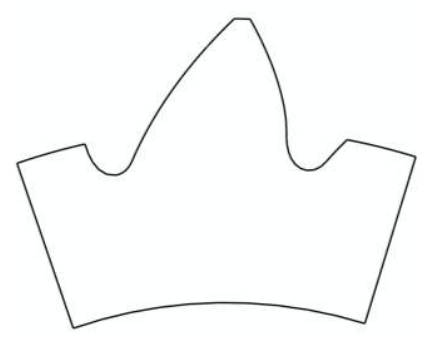

e) Model 5 with pressure angles $40^{\circ} / 20^{\circ}$

Figure 3. Five asymmetric gear models with modified pressure angles $\left(20^{\circ}, 25^{\circ}, 30^{\circ}\right.$, $35^{\circ}$ and $\left.40^{\circ}\right)$ on the drive side and a normal pressure angle $\left(20^{\circ}\right)$ on the coast side

Table 3. Basic parameters of the five asymmetric gears

\begin{tabular}{lllll}
\hline Module & $\begin{array}{l}\text { Number } \\
\text { of teeth }\end{array}$ & Pressure angle & $\begin{array}{l}\text { Addendum } \\
\text { coefficient }\end{array}$ & $\begin{array}{c}\text { Tooth } \\
\text { width }\end{array}$ \\
\hline & & $\alpha_{c}=20^{\circ}$ & $B=20$ \\
$m=5 \mathrm{~mm}$ & $z=20$ & $\alpha_{d}=20^{\circ} / 25^{\circ} / 30^{\circ} / 35^{\circ} / 40^{\circ}$ & $h_{a c}^{*}=1$ & $\mathrm{~mm}$ \\
\hline
\end{tabular}




\section{Load Application}

Figure 4 shows the load $F$ applied at HPSTC $M$ which is located on the drive side of the asymmetric tooth. Here, the load $F$ is divided into the tangent load $F_{t}$ and the normal load $F_{n}$. As the transferred power is assumed to be $P=50 \mathrm{~kW}$ and the rotation speed of the gear is assumed to be $n=1000 \mathrm{r} / \mathrm{min}$ in this analysis, the transferred tangent load $F_{t}$ and the normal load $F_{n}$ can then be calculated based on Eq. (14); thus, the exact values are listed in Table 4.

The torque applied at the tooth is:

$$
T=9550 \times \frac{P}{n}
$$

The whole force applied at the tooth is:

$$
F=\frac{T}{B \times r_{M} \times \cos \alpha_{d M}}=\frac{T}{B \times r_{b}}
$$

and the tangent force and normal force applied at the tooth are:

$$
\left\{\begin{array}{l}
F_{t}=F \times \cos \alpha_{F M} \\
F_{n}=F \times \sin \alpha_{F M}
\end{array}\right.
$$

where $B$ is the width of the gear; $z$ is the tooth number of the pinion; $\alpha_{d M}$ is the pressure angle at point $M, \alpha_{d M}=\arctan \left[\tan \left(\arccos \left(\frac{r_{b}}{r+h_{a c}^{*} \times m}\right)\right)-\frac{2 \pi\left(\varepsilon_{d}-1\right)}{z}\right]$; $\alpha_{F M}$ is the load angle at point $M, \alpha_{F M}=i n v \alpha_{d M}+\alpha_{d M}-i n v \alpha_{d}-\frac{\pi}{z}$ and $\varepsilon_{d}$ is the contact ratio on the drive side.

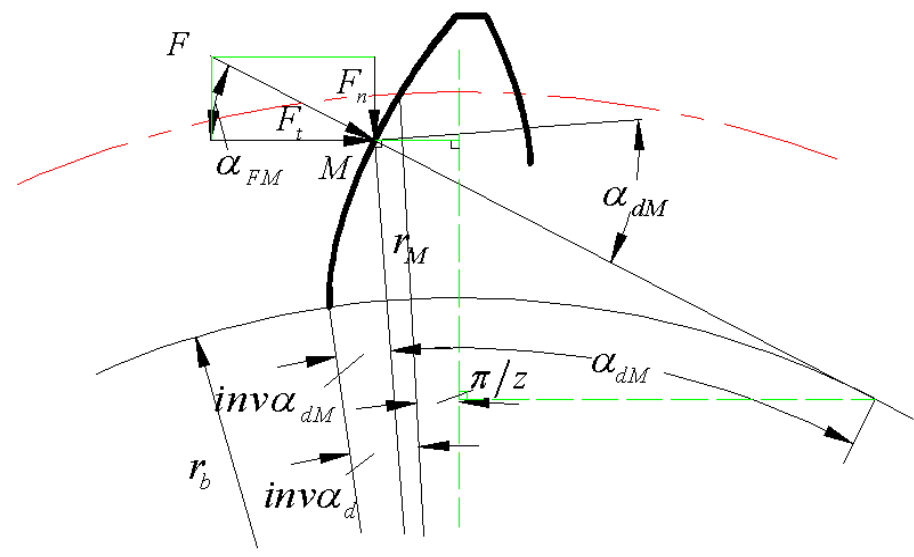

Figure 4. Force applied at the HPSTC on the drive side of the asymmetric tooth 
Table 4. Forces applied at the HPSTC with different asymmetric gear models

Unit: $\mathrm{N} / \mathrm{mm}$

\begin{tabular}{llll}
\hline Pressure angle & Total force $\boldsymbol{F}$ & Tangent force $\boldsymbol{F}_{\boldsymbol{t}}$ & Normal force $\boldsymbol{F}_{\boldsymbol{n}}$ \\
\hline $20^{\circ} / 20^{\circ}$ & 508144.89 & 481551.68 & 162231.99 \\
$25^{\circ} / 20^{\circ}$ & 526862.96 & 476574.41 & 224636.17 \\
$30^{\circ} / 20^{\circ}$ & 551369.51 & 471014.73 & 286624.24 \\
$35^{\circ} / 20^{\circ}$ & 582919.87 & 465552.06 & 350794.59 \\
$40^{\circ} / 20^{\circ}$ & 623331.98 & 460744.50 & 419830.04 \\
\hline
\end{tabular}

\section{COMPUTATIONAL INVESTIGATION OF THE ES-PIM (T3) METHOD}

In this section, the gear model with the pressure angle of $20^{\circ} / 20^{\circ}$, as shown in Figure , was investigated to test the key properties of the present ES-PIM (T3), e.g. the accuracy of stresses, the convergence properties of the strain energy, the convergence rate of the strain energy norm and the computational efficiency.

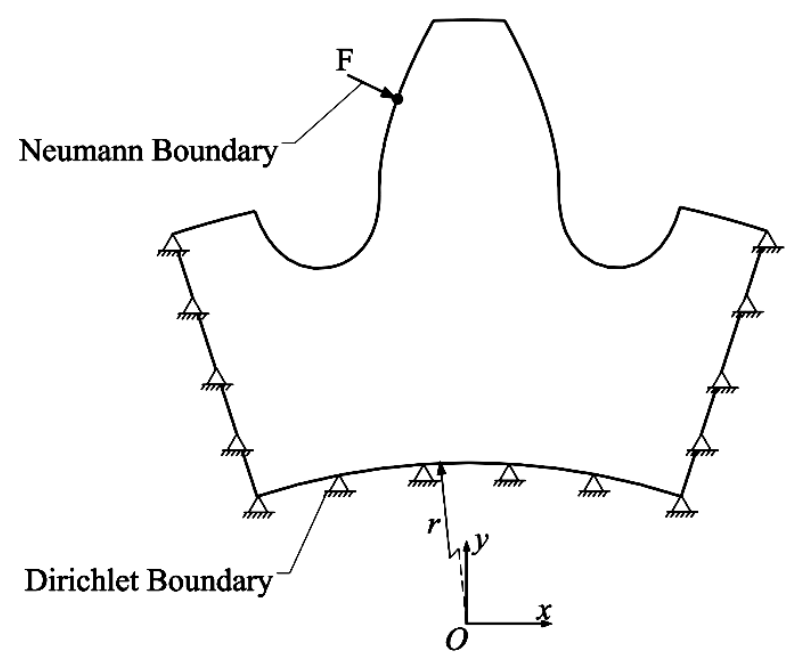

Figure 5. Gear model subjected to Dirichlet and Neumann boundary conditions

An ES-PIM (T3) code developed in MATLAB was used to perform the bending stress analysis. For comparison, the FEM in-house code was also developed here. The properties of the material of the gear were $E=2.16 \times 10^{11} \mathrm{~Pa}$ and $v=0.3$. A mesh with 521 irregularly distributed nodes, as shown in Figure 6a, was generated for the analysis. As the exact stress was not available for the gear problem, a refined mesh with 10,233 irregular nodes (see Figure 6b) was also generated to calculate the reference stress. Note that there as a higher node density at the fillet in order to pay special attention to the stress distribution at that location. The plane strain triangular element was used in this analysis. 


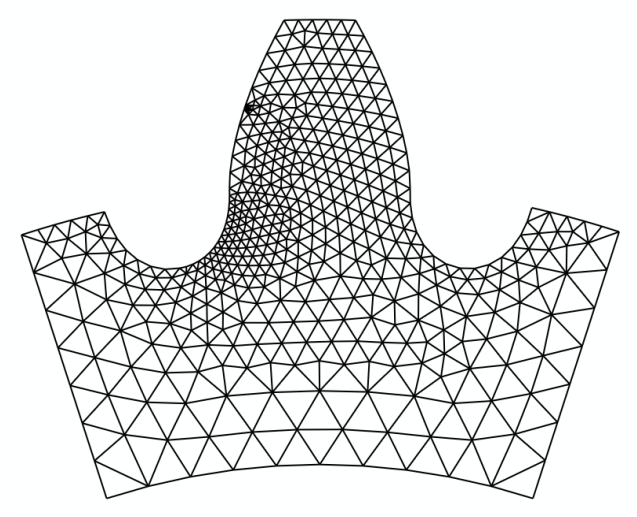

a) Mesh with 521 nodes

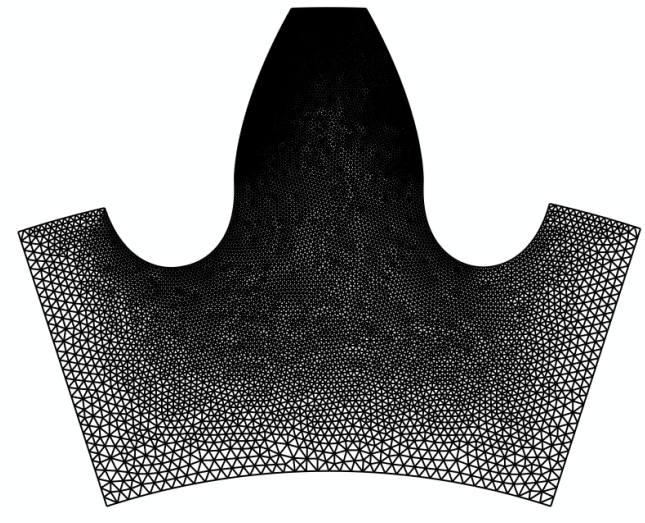

b) Mesh with 10,233 nodes

Figure 4. Meshes generated for analysis

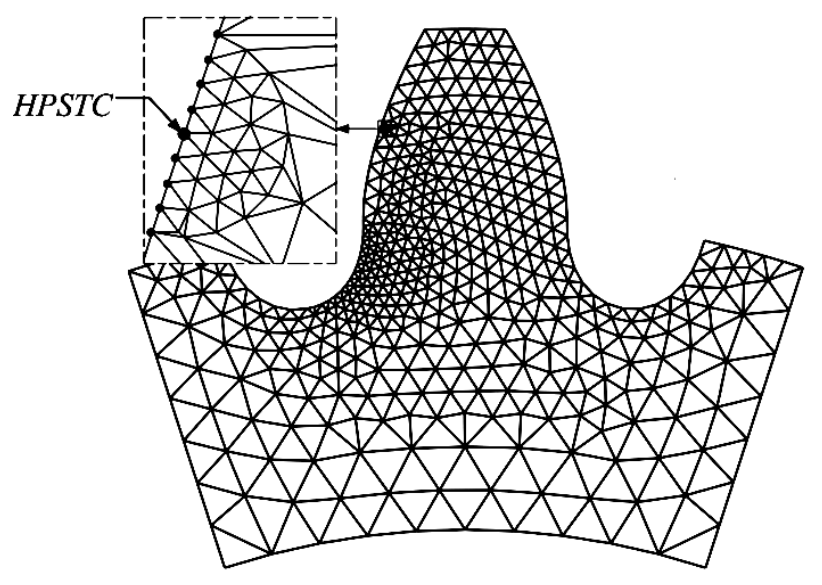

a) Particular mesh near the load point

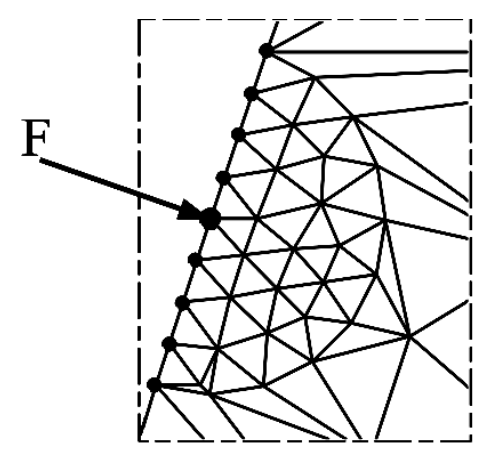

b) Concentrated load applied at the HPSTC
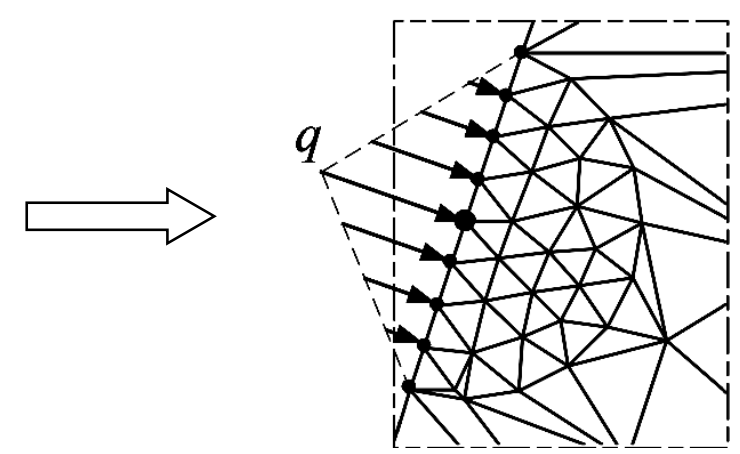

c) Distributed load applied

at the nine points associated with the HPSTC

Figure 5. Load distribution from a concentrated load to a distributed load

The Dirichlet and Neumann boundary conditions were applied at the gear, as shown in Figure 5. For the Dirichlet boundary, the displacements of relevant nodes 
were constrained in both the $X$ and $Y$ directions. For the Neumann boundary condition, the loads of $F_{t}=481551.67 \mathrm{~N}$ and $F_{n}=162231.99 \mathrm{~N}$ were applied at the HPSTC. In order to avoid the infinite value because of the concentrated load, the region near the HPSTC was particularly meshed (Figure 5a); the concentrated load was linearly discretised to nine extremely adjacent nodes on a line associated with the HPSTC using the Gauss integration method, as shown in Figure 5b and Figure 5c.

\section{Accuracy of the Stress Field}

It has been noted that gear teeth usually fail by cracking at the base of the tooth on the tensile-stress side (Wang, 2003). Therefore, it is meaningful to focus on the stress distribution at the fillet of the drive side.

Figure 6 presents the distribution of the von Mises stress at the fillet of the drive side using both the present ES-PIM (T3) and FEM. From these three figures, it can be seen that, at the region near the load point, the stress distribution of the ES-PIM (T3) was much closer to the reference distribution, which means that the ES-PIM (T3) can deal with a concentrated load problem better than the FEM. The distribution of von Mises stresses from the solutions of the present ES-PIM (T3), the FEM and the reference mesh are shown in Figure 7. Figure 8 presents the curves of the stress distributions. From a comparison of the curves in these two figures, it can be seen that the present ES-PIM (T3) solution was much closer to the reference solution compared to the FEM, which means that the ES-PIM (T3) solution was more accurate than that obtained from the FEM computation. Considering the maximum von Mises stress, a $23.67 \%$ reduction in the error was found with the ES-PIM (T3) compared to the FEM.

\section{Convergence of Strain Energy}

The strain energy indicator of a whole domain is defined as follows:

$$
e_{\text {exact }}=\frac{1}{2} \int_{\Omega} \boldsymbol{\sigma}_{\text {exact }}{ }^{\mathrm{T}} \boldsymbol{\varepsilon}_{\text {exact }} \mathrm{d} \Omega=\frac{1}{2} \int_{\Omega} \boldsymbol{\sigma}_{\text {exact }}{ }^{\mathrm{T}} \mathbf{D}^{-1} \boldsymbol{\sigma}_{\text {exact }} \mathrm{d} \Omega
$$

where $\boldsymbol{\sigma}_{\text {exact }}$ is the exact stress vector of an arbitrary point of the domain; $\boldsymbol{\varepsilon}_{\text {exact }}$ is the exact strain vector corresponding to the arbitrary point and $\mathbf{D}$ is the material constant matrix.

Five sets of mesh (321 nodes, 521 nodes, 1102 nodes, 1984 nodes and 4225 nodes) were generated in this subsection for analysis. As the exact strain energy cannot be directly obtained for the gear problem with complex outlines, a reference strain energy based on a refined mesh of 10,233 nodes, which will replace $e_{\text {exact }}$ in Eq. (24), was also calculated. Table 5 lists the strain energies of the present ES-PIM (T3), the FEM method and the reference solution. Figure 9 shows the convergence process of the solutions with an increase in the DOFs. From this figure, it can be seen that i) the strain energies of both the ES-PIM (T3) and FEM converge to the reference solution with an 
increase in the DOFs; ii) the strain energy of the ES-PIM (T3) is much closer to the reference solution than that of FEM. It is well-known that the linear FEM model shows overly stiff behaviour and hence gives poor stress solutions. A softening effect has been introduced into the ES-PIM (T3) owing to the implementation of the strain smoothing operation, which makes it behave more softly and hence obtains more accurate results than the FEM model (Liu and Zhang, 2008).

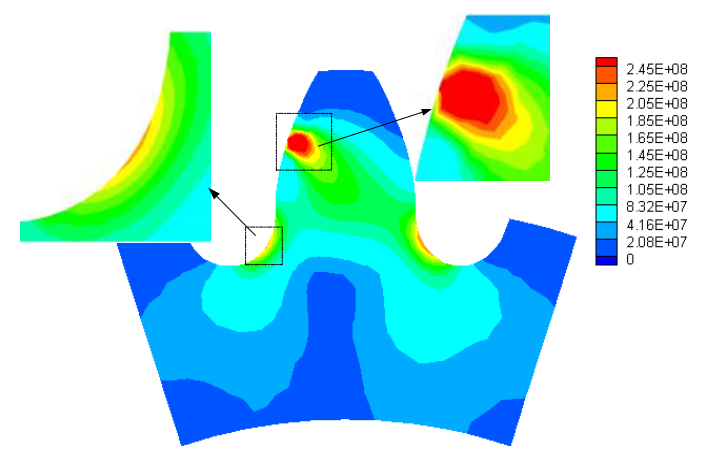

a) ES-PIM (T3) solutions with 521irregular nodes

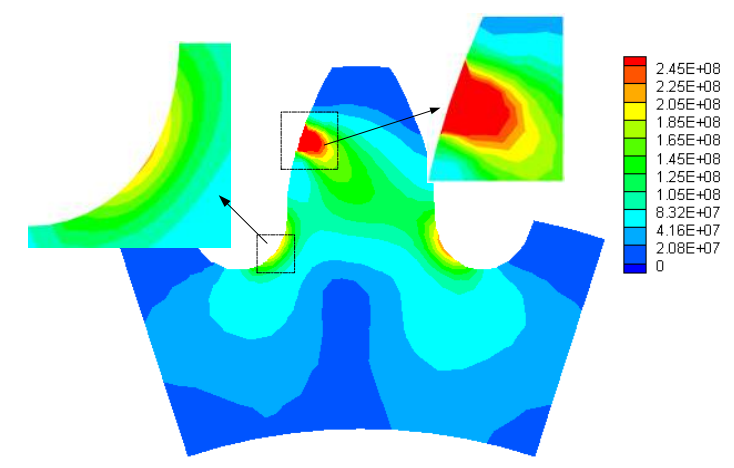

b) FEM solutions with 521 irregular nodes

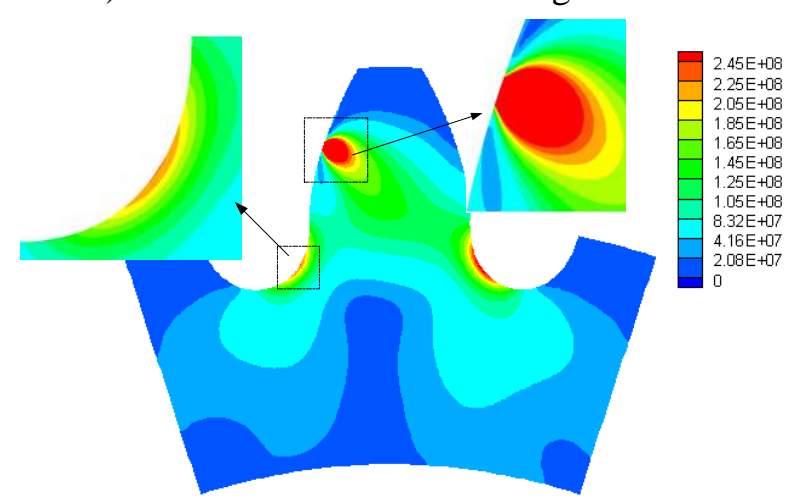

c) Reference solutions with 10,233 irregular nodes

Figure 6. Rainbow figures of the von Mises stress from ES-PIM (T3), FEM and reference solutions 


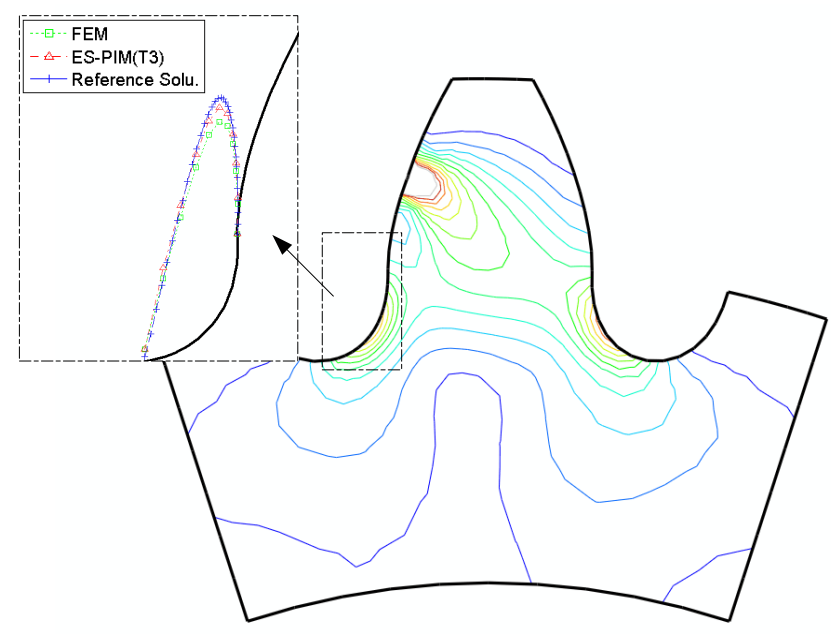

Figure 7. Sketch map of the von Mises stress at the fillet based on the present ES-PIM (T3) and FEM

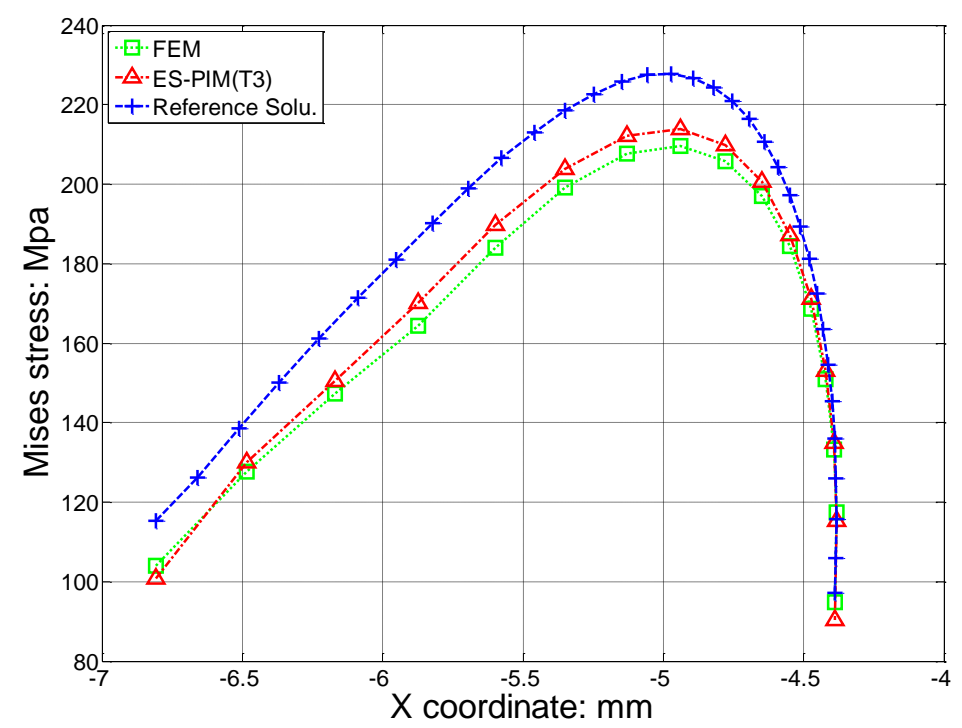

Figure 8. Comparisons of the von Mises stress at the fillet based on the present ES-PIM (T3) and FEM

Table 5. Strain energy with different DOFs

\begin{tabular}{llll}
\hline Case & DOF & Strain energy of ES-PIM (T3) & Strain energy of FEM \\
\hline 1 & 642 & 4.1680 & 3.9507 \\
2 & 1042 & 4.2037 & 4.0429 \\
3 & 2204 & 4.2945 & 4.1925 \\
4 & 3968 & 4.3157 & 4.2385 \\
5 & 8450 & 4.3459 & 4.2920 \\
\multicolumn{2}{l}{ Reference } & $4.3591 \quad$ (20466 DOFs) \\
\hline
\end{tabular}




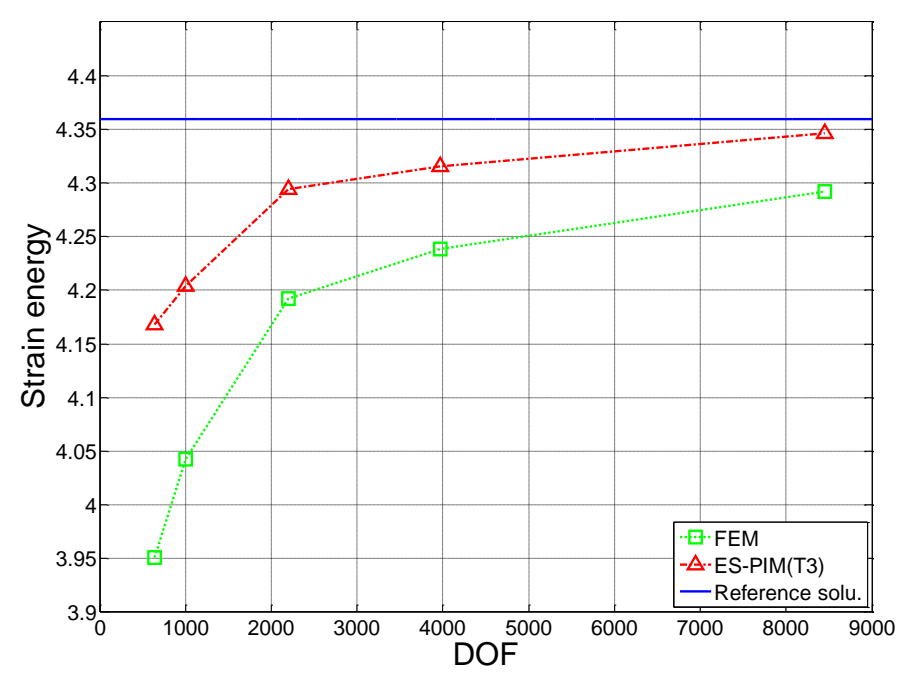

Figure 9. Solutions (in strain energy) converging to the exact solution for the gear using both ES-PIM (T3) and FEM

\section{Convergence Rate of Strain Energy Norm}

The strain energy error norm indicator is defined as follows:

$$
e_{e}=\left(\frac{e_{\text {exact }}-e_{\text {num }}}{e_{\text {exact }}}\right)^{1 / 2}
$$

where $e_{\text {exact }}$ is the exact strain energy of the gear model obtained by Eq. (16) and $e_{\text {num }}$ is the numerical strain energy of the gear model. Here, the $e_{\text {exact }}$ is also replaced by the reference strain energy from a refined mesh as in section 4.2. The convergence rate in energy error norm, converging with the reducing average nodal spacing $(\mathrm{h})$, is plotted in Figure 10. From this figure, it can be found that the convergence rate of the present ES-PIM (T3) of 0.99 is 1.46 times higher than that of the FEM of 0.68.

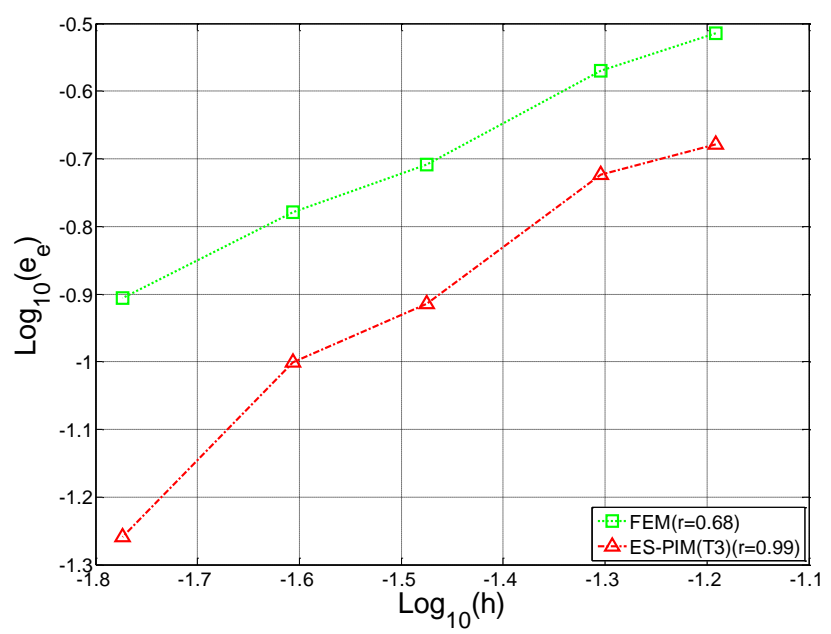

Figure 10. Convergence rate of the numerical results in the energy norm for the gear using both ES-PIM (T3) and FEM 


\section{Computational Efficiency}

A comparison of computational efficiency was investigated on the same computer (Dell PC with an Inter® Pentium(R) $2.80 \mathrm{GHz}$ CPU, 1 GB of RAM) using models with the same DOFs. In order to carry out this study in an efficient manner and to test the computation time with reduced measurement error, a model with four types of irregularly distributed nodes (3010 nodes, 10461 nodes, 30286 nodes and 44407 nodes) was developed and the MFree $2 \mathrm{D}^{\odot}$ was used in a test where a well-coded "bandwidth" solver with an on-column storage technique was available (Liu and Liu, 2003; Wu et al., 2008; Chen et al., 2010). As is known, the computation costs mainly come from two parts: the cost of solving the system equations and the cost of interpolation which mainly solves the inverse of moment matrix to form the shape functions. Table 6 and Table 7 list the CPU time required for these two parts (Chen et al., 2009), respectively. From these two tables, it can be seen that i) the CPU time of ES-PIM (T3) and FEM of interpolation was shorter than that of solving the system equations (especially as the number of nodes became larger) and could be neglected for the whole computation, especially when the model was huge with more DOFs; ii) the CPU time of ES-PIM (T3) for solving the system equations was 2-3.5 times longer than that of FEM. This was mainly because more local nodes were selected by ES-PIM (T3) to approximate the displacement field, which made the bandwidth of the global stiffness matrix two times greater than that of FEM (Wu et al., 2008; Chen et al., 2009). Actually, the CPU time of ES-PIM (T3) for solving the system equations should be four times greater than that of FEM based on bandwidth theory (Chen et al., 2009). Therefore, the test results agree very well with theoretical considerations.

Although more CPU time (2-3.5 times more) was used by ES-PIM (T3), the ES-PIM (T3) method stands out if we consider the computational efficiency (strain energy error norm/CPU time). Tests (Liu and Zhang, 2008; Liu et al., 2008; Chen et al., 2009) have indicated that the solution accuracy of ES-PIM (T3) is much better (by about 10 times) than the linear FEM with the same triangular mesh. Thus, ES-PIM (T3) is a much better choice for the calculation of mechanical problems.

Table 6. Comparison of the CPU time (s) required to solve the system equations by ES-PIM (T3) and FEM with triangular background elements

\begin{tabular}{llll}
\hline Case & DOF & ES-PIM (T3) & FEM \\
\hline 1 & 7020 & 0.359 & 0.156 \\
2 & 20922 & 3.807 & 1.331 \\
3 & 60572 & 23.309 & 9.314 \\
4 & 88814 & 63.391 & 19.22 \\
\hline
\end{tabular}


Table 7. Comparison of the CPU time (s) of interpolation by ES-PIM (T3) and FEM with triangular background elements

Unit: seconds

\begin{tabular}{llll}
\hline Case & DOF & ES-PIM (T3) & FEM \\
\hline 1 & 7020 & 0.281 & 0.267 \\
2 & 20922 & 1.227 & 0.767 \\
3 & 60572 & 4.228 & 2.353 \\
4 & 88814 & 4.478 & 3.622 \\
\hline
\end{tabular}

\section{INVESTIGATION INTO THE STRESS DISTRIBUTION}

The fillet stress distribution on the drive side of an asymmetric gear was investigated in this section. The five asymmetric gear models established in section 3 were utilised. The respective models with their design parameters can be found in

Figure 3 and Table 3. The materials assigned at the models were all linear elastic, with a Young's modulus of $E=2.16 \times 10^{11} \mathrm{~Pa}$ and a Poisson's ratio of $v=0.3$. During the analysis, the five gear models were discretised with approximately irregularly distributed nodes and the equal node space was set on the outline; the node density was higher at the fillet with a node spacing of $0.0003 \mathrm{~mm}$ and was normal in the other parts of the outline with a node spacing $0.0007 \mathrm{~mm}$. Five sets of refined meshes were generated, respectively, for their reference solutions. The node information is listed in Table 8 . The element type, Dirichlet and Neumann boundary conditions are the same as those used in section 4 .

Figure 11 shows the distribution of von Mises stress, and Figure 12 and Figure 13 present the comparisons of these stresses at the fillet. From these figures, it can be seen that i) the solutions from both ES-PIM(T3) and FEM gave the same trends of stress distribution; ii) the maximum bending stress decreased with an increase in the drive side pressure angle; iii) the fillet moved to the negative direction of the $X$-axis and the length of fillet decreased with an increase in the drive side pressure angle and iv) the location of the maximum stress moved to the top of the fillet with an increase in the pressure angle on the drive side. Figure 14 presents a comparison of the maximum bending stress with different pressure angles on the drive side. It was again noted that the maximum bending stress decreased with an increase in the drive side pressure angle. It can also be seen that the stresses calculated by ES-PIM (T3) were much closer to the reference values than those of the FEM, which means that the ES-PIM (T3) should give more accurate solutions than the FEM. The reduced errors were $23.67 \%$, $26.31 \%, 25.90 \%, 25.41 \%$ and $29.73 \%$, respectively, for the present ES-PIM (T3). This conclusion can verify the conclusions found in section 4 . Another conclusion drawn from Figure 14 is that the maximum bending stresses decreased approximately linearly with an increase in the drive side pressure angle. This curve can be synthesised in a straight line with the expression in Eq. (17): 


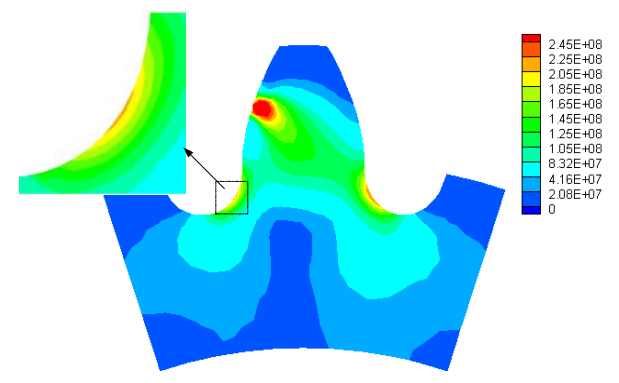

a1) ES-PIM (T3) solutions for the $20^{\circ} / 20^{\circ}$ model

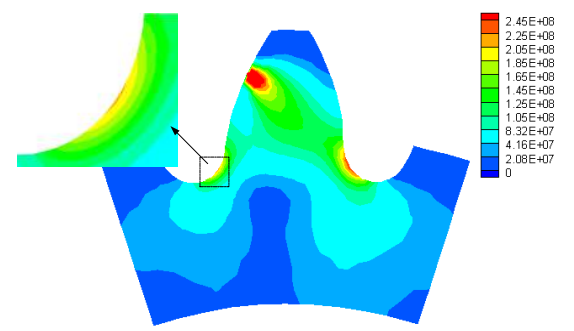

b1) ES-PIM (T3) solutions for the $25^{\circ} / 20^{\circ}$ model

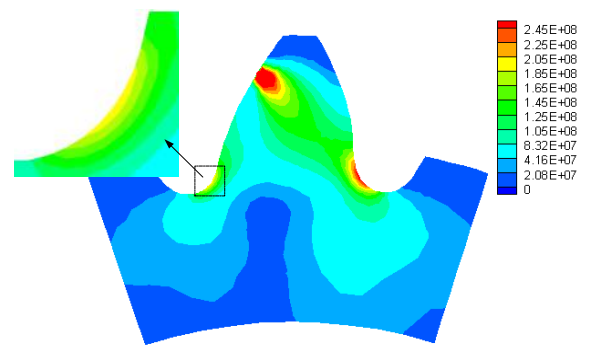

c1) ES-PIM (T3) solutions for the $30^{\circ} / 20^{\circ}$ model

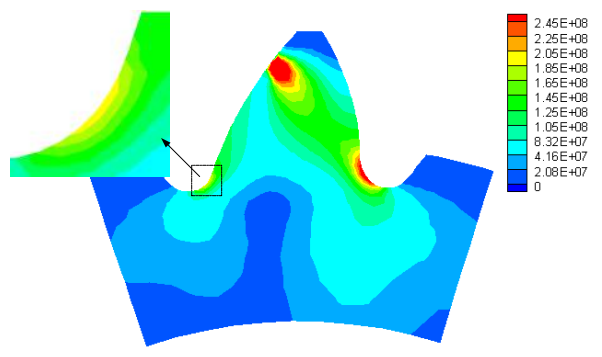

d1) ES-PIM (T3) solutions for the $35^{\circ} / 20^{\circ}$ model

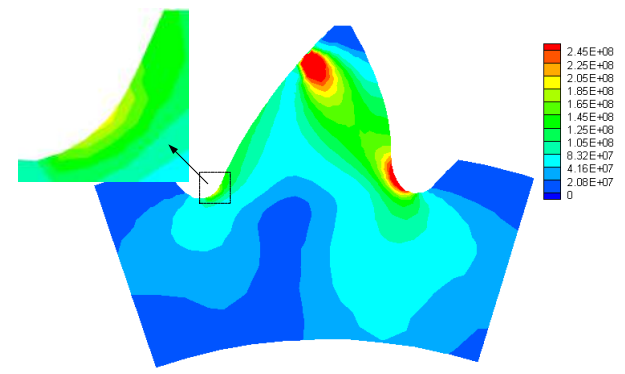

e1) ES-PIM (T3) solutions for $40^{\circ} / 20^{\circ}$ model

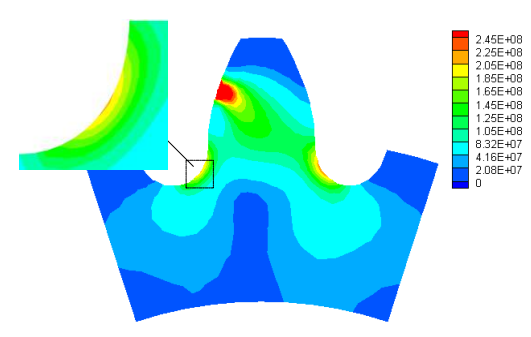

a2) FEM solutions for the $20^{\circ} / 20^{\circ}$ model

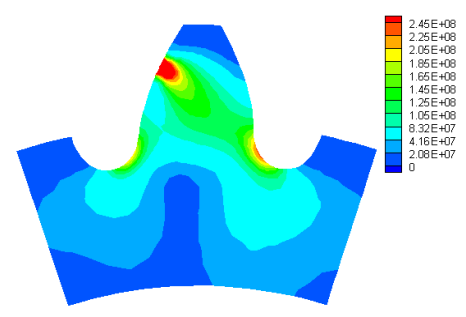

b2) FEM solutions for the $25^{\circ} / 20^{\circ}$ model

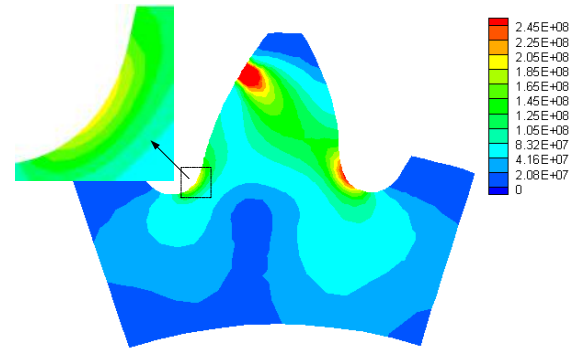

c2) FEM solutions for the $30^{\circ} / 20^{\circ}$ model

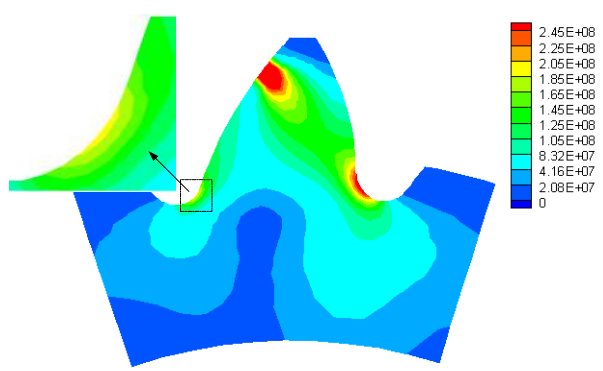

d2) FEM solutions for the $35^{\circ} / 20^{\circ}$ model

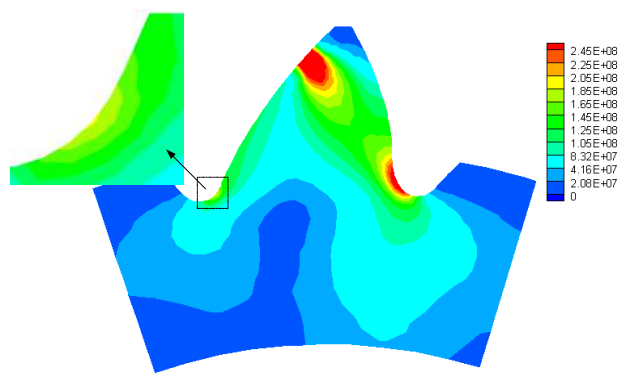

e2) FEM solutions for $40^{\circ} / 20^{\circ}$ model

Figure 11. von Mises stress rainbow of ES-PIM (T3), FEM and reference solutions based on the five asymmetric gear models with pressure angles $20^{\circ} / 20^{\circ}, 25^{\circ} / 20^{\circ}, 30^{\circ} / 20^{\circ}, 35^{\circ} / 20^{\circ}$ and $40^{\circ} / 20^{\circ}$ 


$$
\sigma_{\operatorname{Max}}=-0.877 \alpha_{d}+245.22
$$

where $\alpha_{d}$ is the pressure angle on the drive side and $\sigma_{\operatorname{Max}}$ is the maximum bending stress corresponding to $\alpha_{d}$ in MPa.

Table 8. Node information of the five asymmetric gear models

\begin{tabular}{lcc}
\hline Gear model & Nodes numbers for comparison & Node number for reference \\
\hline $20^{\circ} / 20^{\circ}$ & 521 & 10233 \\
$25^{\circ} / 20^{\circ}$ & 524 & 11100 \\
$30^{\circ} / 20^{\circ}$ & 519 & 11061 \\
$35^{\circ} / 20^{\circ}$ & 510 & 10919 \\
$40^{\circ} / 20^{\circ}$ & 500 & 10928 \\
\hline
\end{tabular}

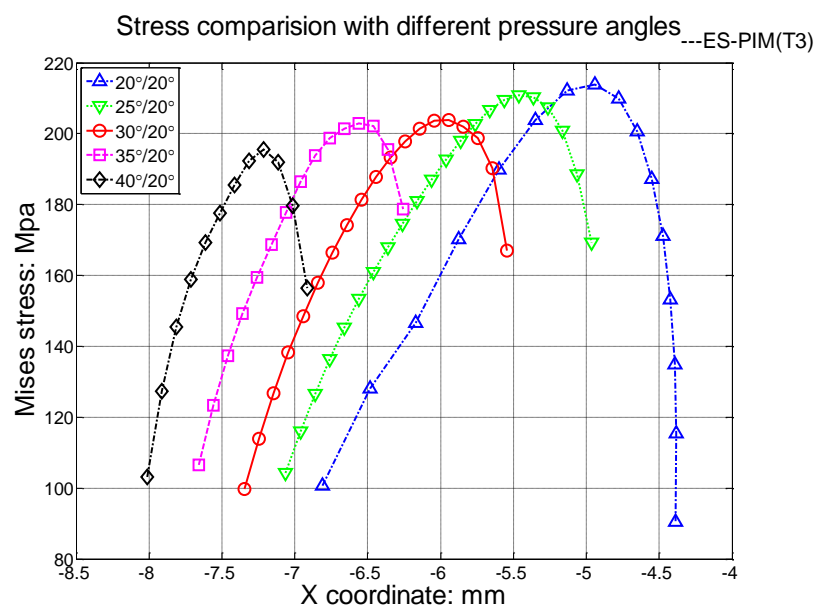

Figure 12. Stress distributions at the fillet of the five asymmetric gear models based on ES-PIM (T3)

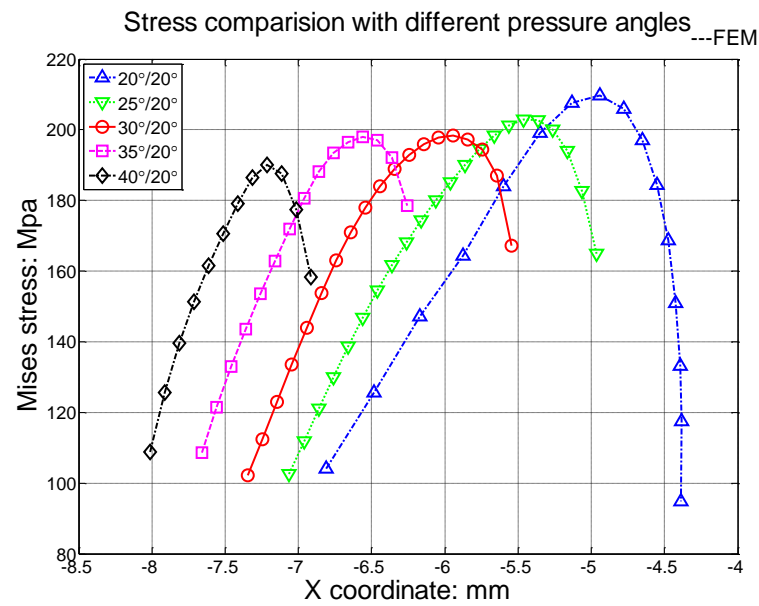

Figure 13. Stress distributions at the fillet of the five asymmetric gear models based on FEM 


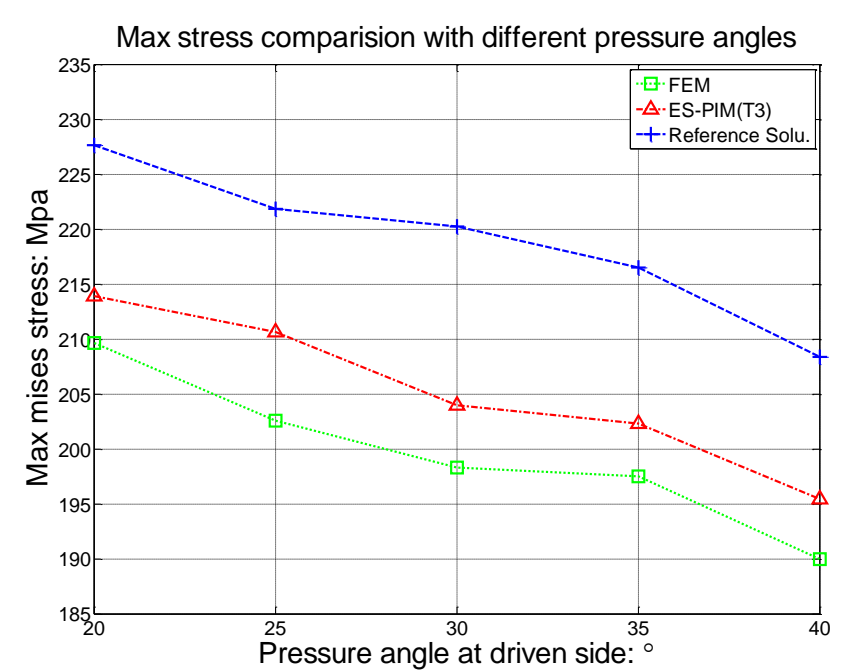

Figure 14. Maximum bending stress comparison based on the present ES-PIM (T3) and FEM using the same coarse triangular elements

\section{CONCLUSIONS}

In this work, newly designed asymmetric gears which use modified pressure angles on the drive side and a standard pressure angle on the coast side were proposed, and the ES-PIM (T3) method using the background mesh of triangular cells was used to conduct asymmetric gear bending stress analysis. Through the formulation and numerical examples, it can be concluded that ES-PIM (T3) possesses significant advantages over the FEM in problem analysis with very complex geometries. More accurate solutions in the stress field were drawn by the ES-PIM (T3) than by FEM. Higher convergence, a higher convergence rate of the strain energy error and a higher computational efficiency were also obtained by the ES-PIM (T3) in this analysis. The problem with stress concentration can be better solved by the present method. With the attractive properties of good accuracy, "super-convergence" and high computational efficiency, the ES-PIM (T3) is very convenient for bending strength analysis of complicated asymmetric gears, which involves complex outlines, stress concentration at the fillet and concentration load at the HPSTC.

\section{ACKNOWLEDGEMENTS}

This work is partially supported by A*Star, Singapore. It was also partially supported by the Open Research Fund Programme of the State Key Laboratory of Advanced Technology of Design and Manufacturing for Vehicle Bodies, Hunan University, P. R. China under the grant number 40915001. 


\section{REFERENCES}

Bible, G.D., Reddy, S.K., Savage, M. and Handschuh, R.F. 1994. Effects of rim thickness on spur gear bending stress. Journal of Mechanical Design, 116: 1157-1162.

Cavdar, K., Karpat, F. and Babalik, F.C. 2005. Computer aided analysis of bending strength of involute spur gears with asymmetric profile. Journal of Mechanical Design, 127: 477-484.

Chen, L., Nguyen, H.X., Nguyen, T.T., Zeng, K.Y. and Wu, S.C. 2010. Assessment of smoothed point interpolation methods for elastic mechanics. International Journal for Numerical Methods in Biomedical Engineering, 26(2): 1635-1655.

Deng, G., Nakanishi, T. and Inoue, K. 2003. Bending load capacity enhancement using an asymmetric tooth profile. JSME International Journal. Series C, Mechanical Systems, Machine Elements and Manufacturing, 46: 1171-1176.

Fetvaci, C. and Imrak, E. 2008. Mathematical model of a spur gear with asymmetric involute teeth and its cutting simulation. Mechanics Based Design of Structures and Machines, 36: 34-46.

Kapelevich, A.L. 2000. Geometry and design of involute spur gears with asymmetric teeth. Mechanism and Machine Theory, 35: 117-130.

Kapelevich, A.L. and Kleiss, R.E. 2002. Direct gear design for spur and helical involute gears. Gear Technology, 19: 29-35.

Kumar, V.S., Muni, D.V. and Muthuveerappan, V.G. 2008. Optimization of asymmetric spur gear drives to improve the bending load capacity. Mechanism and Machine Theory, 43: 829-858.

Litvin, F.L., Lian, Q. and Kapelevich, A.L. 2000. Asymmetric modified gear drives: reduction of noise, localization of contact, simulation of meshing and stress analysis. Computer Methods in Applied Mechanics and Engineering, 188: 363-390.

Liu, G.R. 2002. Meshfree methods: Moving Beyond the Finite Element Method. Boca Raton: CRC Press.

Liu, G.R. 2008a. A generalized gradient smoothing technique and the smoothed bilinear form for Galerkin formulation of a wide class of computational methods. International Journal of Computation Methods, 5: 199-236.

Liu, G.R. 2010. A weakened weak $\left(\mathrm{W}^{2}\right)$ form for a unified formulation of compatible and incompatible methods, Part I: Theory and Part II: Applications to solid mechanics problems. International Journal for Numerical Methods in Engineering, 81(9): 1127-1156.

Liu, G.R. and Gu, Y.T. 2001. A point interpolation method for two-dimensional solids. International Journal for Numerical Methods in Engineering, 50: 937-951.

Liu, G.R. and Liu, M.B. 2003. Smoothed Particle Hydrodynamics-A Meshfree Particle Method. Singapore: World Scientific. 
Liu, G.R. and Quek, S.S. 2003. Finite Element Method: a practical course. Burlington: Butterworth-Heinemann.

Liu, G.R. and Zhang, G.Y. 2008. Edge-based smoothed point interpolation methods. International Journal of Computation Methods 5: 621-646.

Liu, G.R., Nguyen, T.T. and Lam, K.Y. 2008. An edge-based smoothed finite element method (ES-FEM) for static, free and forced vibration analyses of solids. Journal of Sound and Vibration 320: 1100-1130.

Liu, G.R., Zhang, G.Y., Dai, K.Y., Wang, Y.Y., Zhong, Z.H., Li, G.Y. and Han, X. 2005. A linearly conforming point interpolation method (LC-PIM) for 2D mechanics problems. International Journal of Computation Methods, 2: 645-665.

Muni, D.V., Kumar, V.S. and Muthuveerappan, V.G. 2007. Optimization of asymmetric spur gear drives for maximum bending strength using direct gear design method. Mechanics Based Design of Structures and Machines, 35: 127-145.

Su, X.G. and Houser, D.R. 2000. Characteristics of trochoids and their application to determining gear teeth fillet shapes. Mechanism and Machine Theory, 35: 291-304.

Wang, J.G. and Liu, G.R. 2002. A point interpolation meshless method based on radial basis functions. International Journal for Numerical Methods in Engineering, 54: $1623-1648$.

Wang, M.J. 2003. A new photoelastic investigation of the dynamic bending stress of spur gears. Journal of Mechanical Design, 125: 365-372.

Wu, S.C., Liu, G.R., Zhang, H.O., Xu, X. and Li, Z.R. 2008. A node-based smoothed point interpolation method (NS-PIM) for three-dimensional hear transfer problems. International Journal of Thermal Sciences, 48: 1367-1376.

Xiao, W.Q. 2008. Investigation on meshing theory and load capacity of high performance unsymmetrical gear with double pressure angles. Ph.D. dissertation, University of Science and Technology, Beijing.

Xiao, W.Q., Li, W. and Han, J.Y. 2008. Meshing theory on unsymmetric gear with double pressure angles. Transactions of the Chinese Society of Agricultural Machinery, 39: 169-173.

Xiao, W.Q., Li, W. and Li, M. 2006. Finite element analysis of the tooth root bending stress of an unsymmetric gear with double pressure angles. Journal of University of Science and Technology Beijing, 28: 570-575. 